\title{
Article \\ Coadjoint Orbits of the Poincaré Group for Discrete-Spin Particles in Any Dimension
}

\author{
Ismael Ahlouche Lahlali, Nicolas Boulanger *(D) and Andrea Campoleoni ${ }^{+}$(D) \\ Physique de l'Univers, Champs et Gravitation, Université de Mons-UMONS, 20 Place du Parc, \\ B-7000 Mons, Belgium; Ismael.AHLOUCHELAHLALI@student.umons.ac.be (I.A.L.); \\ Andrea.campoleoni@umons.ac.be (A.C.) \\ * Correspondence: nicolas.boulanger@umons.ac.be \\ + Research Associate of the Fund for Scientific Research—FNRS, Belgium.
}

check for updates

Citation: Ahlouche Lahlali, I.; Boulanger, N.; Campoleoni, A. Coadjoint Orbits of the Poincaré Group for Discrete-Spin Particles in Any Dimension. Symmetry 2021, 13, 1749. https://doi.org/10.3390/ sym13091749

Academic Editor:Loriano Bonora

Received: 14 July 2021

Accepted: 18 August 2021

Published: 19 September 2021

Publisher's Note: MDPI stays neutral with regard to jurisdictional claims in published maps and institutional affiliations.

Copyright: (c) 2021 by the authors. Licensee MDPI, Basel, Switzerland. This article is an open access article distributed under the terms and conditions of the Creative Commons Attribution (CC BY) license (https:// creativecommons.org/licenses/by/ $4.0 /)$.

\begin{abstract}
Considering the Poincaré group $\operatorname{ISO}(d-1,1)$ in any dimension $d>3$, we characterise the coadjoint orbits that are associated with massive and massless particles of discrete spin. We also comment on how our analysis extends to the case of continuous spin.
\end{abstract}

Keywords: coadjoint orbit method; unitary irreducible representations of the Poincaré group

\section{Introduction}

The Unitary Irreducible Representations (UIRs) of the Poincaré group in four dimensions $\operatorname{ISO}(3,1)$ were classified by Wigner in his seminal paper [1]. Later, with Bargmann in [2] they associated, with every $\operatorname{UIR}$ of $\operatorname{ISO}(3,1)$, a linear and manifestly relativistic field equation the solutions of which transform in the corresponding UIR. Concerning the UIRs of the Poincaré group in dimensions $d>3$, the first classification was obtained by Siegel and Zwiebach in [3,4]. The three-dimensional case had been treated in [5]. By following a different approach that generalises to any dimension, the Bargmann-Wigner treatment [2], a classification of linear and manifestly relativistic field equations giving rise to a classification of the UIRs of the Poincaré group in arbitrary dimension was obtained in [6-8]. For a pedagogical review of these classifications and their equivalence (together with related works including the recent paper [9] that uses what one could call the "Casimir approach"), see the lecture notes in [10].

Yet another way to study the UIRs of the Poincaré group in any dimension is via Kirillov's coadjoint orbit method [11], although the Poincare group is neither nilpotent nor compact. The coadjoint orbits of the Poincaré group in any dimensions have been classified in the mathematical literature [12]. Still, a physicist-friendly approach appears desirable to us, with a particular focus on the orbits that correspond to massive and massless particles with discrete spin or helicity. In four dimensions, this was done in [13] for the massive case and in the papers [14,15] by Andrzejewski, Kosiński, Maślanka and collaborators for massive and massless particles of discrete spin, with a review presented in [16]. The original scope of the latter analysis was to reconstruct the dynamics of spinning particles directly from Poincaré invariance by identifying the appropriate coadjoint orbit of the Poincaré group with the phase space associated with a given spinning particle. In the current paper, we want to pursue the study of coadjoint orbits associated with massive and massless particles and push it to the Poincaré group for Minkowski spacetime in any dimension $d>3$. The coadjoint orbit method was already applied to the groups $\operatorname{ISO}(2,1)$ and $S O(2,2)$ and various infinite-dimensional extensions of them in, e.g., [17-20]. We refer to these works and to [21-23] for another illustration of the interest in the study of coadjoint orbits of a Lie group $G$, even in the cases where the classification of the UIRs of $G$ is already known. The interest precisely lies in the connection between the coadjoint orbits on the one hand and the UIRs of the Lie group on the other hand. 
In the rest of this introduction, we recall some elementary notions that establish our notation and give definitions that are necessary for the following sections.

First of all, let us recall that, given a Lie group $G$ and its Lie algebra $\mathfrak{g}$ of dimension $n$, the adjoint representation of $G$ is $A d: G \rightarrow A u t(\mathfrak{g})$ such that $G \ni g \mapsto A d_{g}$ where $A d_{g}(x):=$ $g x g^{-1}$, for all $x \in \mathfrak{g}$. Introducing the dual algebra $\mathfrak{g}^{*}$ with the pairing $\left\langle\theta^{I}, e_{J}\right\rangle=\delta_{I}^{I}$ between a basis $\left(e_{I}\right)_{I=1, \ldots, n}$ of $\mathfrak{g}$ and the dual basis $\left(\theta^{I}\right)_{I=1, \ldots, n}$ of $\mathfrak{g}^{*}$, the coadjoint representation of $G$ is defined by $A d^{*}: G \rightarrow A u t\left(\mathfrak{g}^{*}\right)$ such that $G \ni g \mapsto A d_{g}^{*}$ where $\left\langle A d_{g}^{*}(\eta), x\right\rangle=$ $\left\langle\eta, A d_{g^{-1}}(x)\right\rangle$ for all $x \in \mathfrak{g}$ and for all $\eta \in \mathfrak{g}^{*}$.

The Poincaré group $I S O(d-1,1) \cong S O(d-1,1) \ltimes T_{d}$ is the relevant group for relativistic physics in Minkowski spacetime of dimension $d$. It is the semi-direct product of the group of (proper) Lorentz transformations with the abelian group of spacetime translations. The elements of $S O(d-1,1)$ have a unit determinant and preserve the Minkowski metric $\eta$; i.e., for any $\Lambda \in S O(d-1,1)$ one has $\Lambda^{t} \eta \Lambda=\eta$ and $\operatorname{det}(\Lambda)=1$. We work in inertial coordinates where $\eta=\operatorname{diag}(+1,-1, \ldots,-1)$. In the following, we implicitly restrict the proper Lorentz group $S O(d-1,1)$ to its subgroup of orthochronous transformations, where $\Lambda_{0}^{0} \geqslant 1$ on top of the previous constraints. The multiplication law in the Poincaré group is defined as $\left(\Lambda_{1}, a_{1}\right)\left(\Lambda_{2}, a_{2}\right)=\left(\Lambda_{1} \Lambda_{2}, \Lambda_{1} a_{2}+a_{1}\right)$, while the inverse is given by $(\Lambda, a)^{-1}=\left(\Lambda^{-1},-\Lambda^{-1} a\right)$. An infinitesimal Lorentz transformation is given by $\Lambda_{B}^{A}=\delta^{A}{ }_{B}+\omega^{A}{ }_{B}$ where $\omega_{A B}$ is an infinitesimal antisymmetric tensor and the indices $A, B, C, \ldots$ run over the values $0,1, \ldots, d-1$. An element $g \in I S O(d-1,1)$ close to the identity is presented by

$$
g=1+i \epsilon^{A} P_{A}-\frac{i}{2} \omega^{A B} M_{A B},
$$

where the factors $i$ and the signs are purely conventional. Here, $P_{A}$ and $M_{A B}$ are the generators of the Poincaré group, meaning that our basis for $\mathfrak{g}$ is $\left(e_{I}\right)_{I=1, \ldots, n}=\left(P_{A}, M_{A B}\right)$. The dimension of the algebra $\mathfrak{g} \cong i s o(d-1,1)$ is $n=\frac{d(d+1)}{2}$. Our conventions for the commutators of the generators of the Poincaré group are given by

$$
\begin{aligned}
{\left[P_{A}, P_{B}\right] } & =0, \\
{\left[M_{A B}, P_{C}\right] } & =i\left(\eta_{B C} P_{A}-\eta_{A C} P_{B}\right), \\
{\left[M_{A B}, M_{C D}\right] } & =i\left(\eta_{A D} M_{B C}+\eta_{B C} M_{A D}-\eta_{A C} M_{B D}-\eta_{B D} M_{A C}\right) .
\end{aligned}
$$

If a generic element $x \in \mathfrak{g}$ is written as $x=\epsilon^{I} e_{I}=\epsilon^{A} P_{A}+\frac{1}{2} \mathscr{\omega}^{A B} M_{A B}$, in the dual algebra $\mathfrak{g}^{*}$ of the Poincare group, we have $\eta=\zeta_{I} \theta^{I} \in \mathfrak{g}^{*}$ and $\left(\zeta_{I}\right)=\left(\zeta_{A}, \zeta_{A B}\right) \in\left(\mathfrak{g}^{*}\right)^{*} \cong \mathfrak{g}$ are the coordinates of a generic element $\eta \in \mathfrak{g}^{*}$. The dual space is equipped with the following Poisson structure:

$$
\begin{aligned}
\left\{\zeta_{A}, \zeta_{B}\right\} & =0 \\
\left\{\zeta_{A B}, \zeta_{C}\right\} & =\eta_{B C} \zeta_{A}-\eta_{A C} \zeta_{B}, \\
\left\{\zeta_{A B}, \zeta_{C D}\right\} & =\eta_{A D} \zeta_{B C}+\eta_{B C} \zeta_{A D}-\eta_{A C} \zeta_{B D}-\eta_{B D} \zeta_{A C}
\end{aligned}
$$

\section{Massive Case in Five Dimensions}

The Poincaré algebra in 5 dimensions contains 15 generators: 5 translations collected in $P_{A}$ and 10 Lorentz transformations collected in the antisymmetric tensor $M_{A B}$. The commutation relations are those recalled in Equation (2), and there are three Casimir operators:

$$
C_{1}:=P_{A} P^{A}, \quad C_{2}:=\mathbb{W}^{A B} \mathbb{W}_{A B}, \quad C_{3}:=\mathbb{H}=\mathbb{W}^{A B} M_{A B},
$$

where $\mathbb{W}^{A B}$ is the analogue of the four-dimensional Pauli-Lubanski vector, which is defined as

$$
\mathbb{W}^{A B}:=\frac{1}{2} \epsilon^{A B C D E} M_{C D} P_{E}
$$

In this section, capital Latin indices take values in $\{0,1, \ldots, 4\}$. 
Irreducible massive representations of $I S O(4,1)$ are characterised by the following conditions:

$$
\begin{aligned}
P_{A} P^{A} & =m^{2} \mathbb{I}, \\
\frac{1}{8}\left(\mathbb{W}^{A B} \mathbb{W}_{A B}+m \mathbb{H}\right) & =-m^{2} s_{1}\left(s_{1}+1\right) \mathbb{I}, \\
\frac{1}{8}\left(\mathbb{W}^{A B} \mathbb{W}_{A B}-m \mathbb{H}\right) & =-m^{2} s_{2}\left(s_{2}+1\right) \mathbb{I} .
\end{aligned}
$$

The little group of a massive particle in five dimensions is $S O(4)$; that is, the product of two $S U(2)$ factors. The two (half) integers $s_{1}$ and $s_{2}$ thus label the irreducible representations of the two $S U(2)$ subgroups of $S O(4)$. In the dual algebra, as in (3), we denote by $\zeta_{A}$ and $\zeta_{A B}$, respectively, the dual coordinates associated with $P_{A}$ and $M_{A B}$. We also introduce the dual of the Pauli-Lubanski tensor:

$$
\omega^{A B}:=\frac{1}{2} \epsilon^{A B C D E} \zeta_{C D} \zeta_{E} .
$$

The constraints (6) imply the following constraints in the dual algebra:

$$
\begin{aligned}
\zeta_{A} \zeta^{A} & =m^{2}, \\
\frac{1}{8}\left(\omega^{A B} \omega_{A B}+m \omega^{A B} \zeta_{A B}\right) & =-m^{2} s_{1}\left(s_{1}+1\right), \\
\frac{1}{8}\left(\omega^{A B} \omega_{A B}-m \omega^{A B} \zeta_{A B}\right) & =-m^{2} s_{2}\left(s_{2}+1\right) .
\end{aligned}
$$

We are imposing 3 independent constraints on our 15 coordinates, implying that the orbit for a massive particle is 12-dimensional. Actually, with (8), we are imposing the constraint that the three independent Casimir operators are proportional to the identity with a given eigenvalue. As recalled, e.g., in [16], this operation identifies a generic coadjoint orbit of the Poincaré group.

We now wish to find a convenient parametrisation of these orbits in terms of a set of independent coordinates. We shall thus identify a canonical point on the orbit and characterise the whole orbit by acting on it with the Poincare group. To begin with, it is convenient to solve the mass-shell condition (8a) by choosing the coordinate $\zeta_{A}$ as

$$
\zeta_{A}=(m, 0,0,0,0)
$$

which is the momentum of a massive, positive-energy particle in its rest frame. With such a representative for the coordinates $\zeta_{A}$, the non-zero components of the tensor $\omega^{A B}$ defined in (7) are

$\omega^{12}=m \zeta_{34}, \quad \omega^{13}=m \zeta_{42}, \quad \omega^{14}=m \zeta_{23}, \quad \omega^{23}=m \zeta_{14}, \quad \omega^{24}=m \zeta_{31}, \quad \omega^{34}=m \zeta_{12}$

and the last two equations of (8) become:

$$
\begin{aligned}
& \frac{1}{8}\left[\left(\zeta_{34}+\zeta_{12}\right)^{2}+\left(\zeta_{42}+\zeta_{13}\right)^{2}+\left(\zeta_{14}+\zeta_{23}\right)^{2}\right]=s_{1}\left(s_{1}+1\right) \\
& \frac{1}{8}\left[\left(\zeta_{34}-\zeta_{12}\right)^{2}+\left(\zeta_{42}-\zeta_{13}\right)^{2}+\left(\zeta_{14}-\zeta_{23}\right)^{2}\right]=s_{2}\left(s_{2}+1\right) .
\end{aligned}
$$

Defining

$$
\begin{array}{lll}
J_{1}=-\frac{1}{2}\left(\zeta_{34}+\zeta_{12}\right), & J_{2}=-\frac{1}{2}\left(\zeta_{42}+\zeta_{13}\right), & J_{3}=-\frac{1}{2}\left(\zeta_{14}+\zeta_{23}\right), \\
J_{1}^{\prime}=-\frac{1}{2}\left(\zeta_{34}-\zeta_{12}\right), & J_{2}^{\prime}=-\frac{1}{2}\left(\zeta_{42}-\zeta_{13}\right), & J_{3}^{\prime}=-\frac{1}{2}\left(\zeta_{14}-\zeta_{23}\right),
\end{array}
$$


then, Equation (11) gives

$$
\frac{1}{2}\left(J_{1}^{2}+J_{2}^{2}+J_{3}^{2}\right)=s_{1}\left(s_{1}+1\right), \quad \frac{1}{2}\left(J_{1}^{2^{\prime}}+J_{2}^{2^{\prime}}+J_{3}^{2^{\prime}}\right)=s_{2}\left(s_{2}+1\right) .
$$

This reconstructs the Casimir operator of each factor of $S O(4) \cong S U(2) \times S U(2)$.

As we recalled in the Introduction, the commutation relations of the Lie algebra $\mathfrak{g} \cong i s o(4,1)$ endow the dual algebra $\mathfrak{g}^{*}$ with a Poisson structure. The Poisson bracket between the six variables $\left\{J_{i}, J_{i}^{\prime}\right\}$ (where the indices $i, j$ here and below belong to $\{1,2,3\}$ ) read as follows:

$$
\left\{J_{i}, J_{j}^{\prime}\right\}=0, \quad\left\{J_{i}, J_{j}\right\}=-\epsilon_{i j k} J^{k}, \quad\left\{J_{i}^{\prime}, J_{j}^{\prime}\right\}=-\epsilon_{i j k} J^{\prime k} .
$$

Those relations show that the two sets $J_{i}$ and $J_{i}^{\prime}$ are the generators of the two $S U(2)$ factors of $S O(4)$.

We can now complete the identification of the canonical point of the orbit by applying the same reasoning as in the four-dimensional case studied in $[15,16]$, to which we refer readers for more details. The constraints (8) discussed above are indeed compatible with the following choice for the canonical point:

$$
\bar{\zeta}_{A}=(m, 0,0,0,0), \quad \bar{\zeta}_{0 a}=0, \quad \bar{\zeta}_{a b}=\frac{1}{2} S^{c d} \epsilon_{a b c d},
$$

where $a, b, c, \ldots \in\{1,2,3,4\}$ and $S^{a b}$ is an antisymmetric tensor whose components have to obey the constraints in (11). Imposing the constraint that all components of $S^{a b}$ but $S^{12}$ and $S^{34}$ vanish and considering the sum and the difference of Equations (11a) and (11b) yields the following system of constraints on the nonzero components of $S^{a b}$ :

$$
\left(S^{12}\right)^{2}+\left(S^{34}\right)^{2}=s_{1}\left(s_{1}+1\right)+s_{2}\left(s_{2}+1\right), \quad 2 S^{12} S^{34}=s_{1}\left(s_{1}+1\right)-s_{2}\left(s_{2}+1\right) .
$$

We solve this system by

$$
S^{a b}=\left\{\begin{array}{ccl}
0 & \text { if } & (a, b) \neq(1,2),(3,4) \\
\frac{1}{\sqrt{2}}\left(\alpha_{1}-\alpha_{2}\right) & \text { if } & (a, b)=(1,2) \\
\frac{1}{\sqrt{2}}\left(\alpha_{1}+\alpha_{2}\right) & \text { if } & (a, b)=(3,4)
\end{array}\right.
$$

where the real numbers $\alpha_{1}$ and $\alpha_{2}$ are such that $\alpha_{1}^{2}=s_{1}\left(s_{1}+1\right)$ and $\alpha_{2}^{2}=s_{2}\left(s_{2}+1\right)$.

To determine the coordinate of a generic point of the orbit, one must act via the coadjoint action with a generic Poincaré transformation $(\Lambda, a)$ on the canonical point. We use the same type of parametrisation for a generic element of the Poincare group as in [16]. This means that we impose

$$
\Lambda=L R \quad \text { with } \quad R=\bar{R} B ，
$$

where $R$ is a rotation, $L$ a boost and $B$ an element of $S O(4)$ that stabilises the canonical point. The components of $R$ and $L$ are given as

$$
L_{0}{ }^{A} \equiv L_{A}^{0}=\Lambda_{0}^{A}, \quad L_{b}^{a}=\delta_{b}^{a}-\frac{\Lambda^{a}{ }_{0} \Lambda_{b}{ }^{0}}{1+\Lambda_{0}^{0}}, \quad R_{0}{ }^{A} \equiv R_{A}^{0}=\delta_{0}^{A}, \quad R_{b}^{a}=\Lambda_{b}^{a}-\frac{\Lambda_{0}^{a} \Lambda^{0}{ }_{b}}{1+\Lambda_{0}^{0}},
$$

while we take

$$
B=\left(\begin{array}{ccccc}
1 & 0 & 0 & 0 & 0 \\
0 & \cos \alpha & -\sin \alpha & 0 & 0 \\
0 & \sin \alpha & \cos \alpha & 0 & 0 \\
0 & 0 & 0 & \cos \beta & -\sin \beta \\
0 & 0 & 0 & \sin \beta & \cos \beta
\end{array}\right)
$$


Then, we can use the same parametrisation of the proper orthochronous Lorentz group as was done in [16] for $d=4$ :

$$
(\Lambda, a)=(1, y)(L, 0)(\bar{R}, 0)(B, z)=(L \bar{R} B, L \bar{R} z+y),
$$

where $y$ and $z$ are five vectors such that $y^{0}=0$ and $z^{a}=0$. The Poincaré transformation $(B, z)$ stabilises our canonical point. Now, we are able to show how the translations are expressed in this parametrisation:

$$
a^{A}=L_{B}^{A} \bar{R}_{C}^{B} z^{C} .
$$

As recalled above, in order to read off the expression for a generic point of our orbit for a positive-energy massive particle, we act on our canonical point $\left(\bar{\zeta}_{A}, \bar{\zeta}_{A B}\right)$ with the coadjoint action

$$
\begin{aligned}
& A d^{*}{ }_{(\Lambda, a)}\left(\bar{\zeta}_{A}\right)=\Lambda_{A}{ }^{B} \bar{\zeta}_{B}=\Lambda_{A}{ }^{0} m=: p_{A}, \\
& A d^{*}{ }_{(\Lambda, a)}\left(\bar{\zeta}_{0 b}\right)=\Lambda_{0}^{A} \Lambda_{b}^{B} \bar{\zeta}_{A B}-\left(\Lambda_{0}^{A} a_{b}-\Lambda_{b}{ }^{A} a_{0}\right) \bar{\zeta}_{A}, \\
& A d_{(\Lambda, a)}^{*}\left(\bar{\zeta}_{b c}\right)=\Lambda_{b}^{A} \Lambda_{c}^{B} \bar{\zeta}_{A B}-\left(\Lambda_{b}^{A} a_{c}-\Lambda_{c}^{A} a_{b}\right) \bar{\zeta}_{A},
\end{aligned}
$$

which yields

$$
\zeta_{0 b}=-p_{0} y_{b}+\frac{p^{e} S^{c d} \epsilon_{b c d e}}{2 m}, \quad \zeta_{b c}=-p_{b} y_{c}+p_{c} y_{b}+\frac{1}{2} S^{e f} \epsilon_{e f b c}+\frac{S^{e f}\left[p_{b} \epsilon_{c e f d}-p_{c} \epsilon_{b e f d}\right] p^{d}}{2 m\left(m+p^{0}\right)} \text {. }
$$

We can make this clearer by performing the following change of variables:

$$
x_{a}:=y_{a}-\frac{\epsilon_{a b c d} S^{b c} p^{d}}{2\left(m+p^{0}\right)}, \quad J_{a b}:=\frac{1}{2} S^{c d} \epsilon_{a b c d} .
$$

Then, the coordinates parametrising the coadjoint orbit for a massive particle read as follows:

$$
\zeta_{a b}=-p_{a} x_{b}+p_{b} x_{a}+J_{a b}, \quad \zeta_{0 a}=-p_{0} x_{a}-\frac{p^{b} J_{a b}}{m+p^{0}} .
$$

The Poisson structure for the dual algebra of the Poincare group induces the following non-degenerate Poisson structure on the coadjoint orbit, as is guaranteed by a well-known general result (see, e.g., [24]):

$$
\begin{array}{ll}
\left\{J_{a b}, J_{c d}\right\}=\delta_{a c} J_{b d}+\delta_{b d} J_{a c}-\delta_{a d} J_{b c}-\delta_{b c} J_{a d}, & \left\{x_{a}, p_{0}\right\}=\frac{p_{a}}{p_{0}}, \\
\left\{p_{a}, p_{b}\right\}=0, \quad\left\{x_{a}, x_{b}\right\}=0, \quad\left\{x_{a}, p_{b}\right\}=\delta_{a b}, & \left\{x_{a}, J_{b c}\right\}=0, \quad\left\{p_{a}, J_{b c}\right\}=0 .
\end{array}
$$

We can now reconstruct the "quantum" algebra by quantising the system above following the same process as in the $d=4$ case studied in [16]. To this end, we work with the momentum representation and consider wave-functions depending on the momentum coordinate $p^{a}$ and on the little-group quantum numbers of each particle. In the case of scalar wave-functions, we can introduce the scalar product

$$
(f, g)=\int \frac{d^{4} p}{2 p_{0}} \overline{f(p)} g(p),
$$

where $p_{0}$ is the function of the four classical variables $p^{a}$ that solves the mass-shell relation $p_{A} p^{A}=m^{2}$. We refer readers to [10] for the generalisation of the scalar product (28) to wave-functions carrying a non-trivial spin, as we do not need this for our current analysis. Looking at the Poisson bracket (27), we see that the four quantities $x_{a}$ essentially become 
derivative operators with respect to $p_{a}$. However, we should enforce the hermiticity of these four operators with respect to the inner product defined above, which gives

$$
\hat{x}_{a}=i\left(-\frac{\partial}{\partial p^{a}}-\frac{p_{a}}{2\left(p_{0}\right)^{2}}\right) \mathbb{I},
$$

while $S_{a b}$ is turned into the operator $\hat{S}_{a b}$ obeying the commutation relations of $s o(4)$, as is clear from the first equation of (27) and the relation (25) between $S_{a b}$ and $J_{a b}$. We also have to replace $p_{0} x_{a}$ by $\frac{1}{2}\left(\hat{P}_{0} \hat{x}_{a}+\hat{x}_{a} \hat{P}_{0}\right)$ to take care of the ordering of operators. Then, the generators of the Poincaré algebra take the form

$$
\begin{aligned}
\hat{P}_{A} & =p_{A} \mathbb{I} \\
\hat{M}_{0 a} & =-i p_{0} \frac{\partial}{\partial p^{a}} \mathbb{I}-\frac{p^{d} \hat{S}^{b c} \epsilon_{a b c d}}{2\left(m+p^{0}\right)} \\
\hat{M}_{a b} & =-i\left(p_{b} \frac{\partial}{\partial p^{a}}-p_{a} \frac{\partial}{\partial p^{b}}\right) \mathbb{I}+\frac{1}{2} \hat{S}^{c d} \epsilon_{a b c d} .
\end{aligned}
$$

\section{Massless Case in Five Dimensions}

For massless particles, the first two Casimir operators in (4) must vanish and the orbit must be non-generic with a dimension smaller than that of a massive particle. To identify the additional constraints on the orbit, inspired by [9], we characterise massless irreducible representations with fixed helicity by

$$
\begin{aligned}
P_{A} P^{A} & =0, \\
\epsilon_{A B C D E} P^{C} \mathbb{W}^{D E} & =0, \\
\mathbb{S}_{A} & =s(s+1) P_{A},
\end{aligned}
$$

where we have introduced the spin operator

$$
\mathbb{S}_{A}:=-\frac{1}{4} \epsilon_{A B C D E} M^{B C} \mathbb{W}^{D E} .
$$

The constraints (31b) and (31c) can be consistently imposed since

$$
\left[P_{A}, \mathbb{S}_{B}\right]=\frac{i}{2} \epsilon_{A B C D E} P^{C} \mathbb{W} D E
$$

and they both imply $\mathbb{W}_{A B} \mathbb{W}^{A B}=0$, which is readily seen upon contracting the first condition with $M^{A B}$ or the second with $P^{A}$ (in $d=4$, the analogues of the constraints (31) are

$$
P_{A} P^{A}=0, \quad \epsilon_{A B C D} P^{C} \mathbb{W}^{D}=0, \quad \mathbb{S}_{A}=s^{2} P_{A},
$$

where

$$
\mathbb{W}_{A}:=\frac{1}{2} \epsilon_{A B C D} M^{B C} P^{D}, \quad \mathbb{S}_{A}:=\frac{1}{2} \epsilon_{A B C D} M^{B C} \mathbb{W}^{D} .
$$

The second constraint implies $\mathbb{W}_{A} \mathbb{W}^{A}=0$, while $P^{A} \mathbb{W}_{A}=0$ by construction. Being a null vector orthogonal to the null vector $P_{A}$, the Pauli-Lubanski vector $\mathbb{W}_{A}$ must be proportional to the latter. The condition on $\mathbb{S}_{A}$ then fixes the proportionality factor, meaning that one recovers the standard relation $\mathbb{W}_{A}=s P_{A}$ characterising a massless particle with helicity $s$ in $d=4)$. These conditions are translated into the following constraints on $g^{*}$ :

$$
\begin{aligned}
\zeta_{A} \zeta^{A} & =0, \\
\epsilon_{A B C D E} \zeta^{C} \omega^{D E} & =0, \\
-\frac{1}{4} \epsilon_{A B C D E} \zeta^{B C} \omega^{D E} & =s(s+1) \zeta_{A},
\end{aligned}
$$


where the second equation is called the helicity condition [9].

To identify the precise number of independent constraints, we begin by choosing our standard momentum coordinates as $\zeta_{A}=(k, 0,0,0,-k)$. From (7), we find that the non-zero components of the Pauli-Lubanski tensor are

$$
\begin{aligned}
& \omega^{01}=\omega^{41}=-k \zeta_{23}, \quad \omega^{02}=\omega^{42}=-k \zeta_{31}, \quad \omega^{03}=\omega^{43}=-k \zeta_{12}, \quad \omega^{04}=0, \\
& \omega^{12}=k\left(\zeta_{30}+\zeta_{34}\right), \quad \omega^{23}=k\left(\zeta_{10}+\zeta_{14}\right), \quad \omega^{31}=k\left(\zeta_{20}+\zeta_{24}\right) .
\end{aligned}
$$

The helicity condition (34b) then yields

$$
\zeta_{i 0}=-\zeta_{i 4}, \quad i=1,2,3 .
$$

Considering this result, Equation (34c) eventually gives

$$
\left[\left(\zeta_{12}\right)^{2}+\left(\zeta_{31}\right)^{2}+\left(\zeta_{32}\right)^{2}\right]=s(s+1) .
$$

This extra constraint tells us that the Casimir operator of the little group $S O(3)$ of a massless particle with fixed helicity in $d=5$ is constrained to be $s(s+1) \mathbb{I}$, therefore identifying the label $s$ with the helicity of the particle. Finally, we have five independent constraints on the orbit: three of them come from Equation (36), the fourth condition is the mass-shell constraint (34a), while the fifth and last constraint is (37). Altogether, this means that our orbit is $10(=15-5)$ dimensional.

By looking at the coadjoint action of the Poincare group on a generic point of the orbit, we see that we can take $\bar{\zeta}_{0 a}=0$ for our canonical point. The coadjoint action also tells us that $\zeta_{a b}$ has to transform as an antisymmetric tensor under the rotation group $S O(4)$. This means that we can take

$$
\bar{\zeta}_{a b}=\frac{1}{2} S^{c d} \epsilon_{a b c d},
$$

where $S^{a b}$ are the components of an antisymmetric $S O(4)$ tensor. To summarise, the coordinates of our canonical point can be chosen as

$$
\bar{\zeta}_{A}=(k, 0,0,0,-k), \quad \bar{\zeta}_{0 a}=0, \quad \bar{\zeta}_{a b}=\frac{1}{2} S^{c d} \epsilon_{a b c d} .
$$

To find the generic point of the orbit, we use the same type of parametrisation of a generic Poincare element as was done for the $d=4$ massless case in [14]. The only requirement for this parametrisation comes from the decomposition $\Lambda=B D R$ for the Lorentz matrix, where the matrices $D$ and $R$ are in the stability subgroup $G_{S} \subset I S O(4,1)$ of our canonical point. We take the matrices $B, D$ and $R$ as follows, in the light-conecoordinates $\left(x^{+}, x^{-}, x^{i}\right)$ :

$$
B=\left(\begin{array}{ccccc}
\Lambda^{+}+ & 0 & 0 & 0 & 0 \\
\Lambda^{-}+ & \frac{1}{\Lambda^{+}} & \frac{\Lambda^{1}+}{\Lambda^{+}+} & \frac{\Lambda^{2}+}{\Lambda^{+}} & \Lambda^{3} \Lambda_{+}^{+} \\
\Lambda^{1}+ & 0 & 1 & 0 & 0 \\
\Lambda^{2}{ }_{+} & 0 & 0 & 1 & 0 \\
\Lambda^{3}{ }_{+} & 0 & 0 & 0 & 1
\end{array}\right), \quad D=\left(\begin{array}{ccccc}
1 & \frac{\Lambda^{+}-}{\Lambda^{+}} & d_{1} & d_{2} & d_{3} \\
0 & 1 & 0 & 0 & 0 \\
0 & d_{1} & 1 & 0 & 0 \\
0 & d_{2} & 0 & 1 & 0 \\
0 & d_{3} & 0 & 0 & 1
\end{array}\right), \quad R=\left(\begin{array}{ccccc}
1 & 0 & 0 & 0 & 0 \\
0 & 1 & 0 & 0 & 0 \\
0 & 0 & b_{1} & b_{2} & b_{3} \\
0 & 0 & c_{1} & c_{2} & c_{3} \\
0 & 0 & e_{1} & e_{2} & e_{3}
\end{array}\right)
$$

One can readily determine the expressions for the four three-vectors $b_{i}, c_{i}, d_{i}$ and $e_{i}$ in term of the entries of $\Lambda$ by computing $\Lambda=B D R$. We do not need these expressions. Then, a generic element of the Poincaré group can be written as

$$
(\Lambda, a)=(B, y)(D R, h),
$$

where the matrix $D R$ and the five-vector $h$ are elements of $G_{s}$. In the original Minkowskian coordinates $x^{A}=\left(x^{0}, x^{a}\right)$, the five-vector $y$ has a vanishing time component: $y^{0}=0$. Then, the coadjoint action of the Poincaré group on our canonical point for a massless particle 
in $d=5$ gives the following coordinates for a generic point of the coadjoint orbit, in an arbitrary inertial coordinate system:

$$
\begin{aligned}
& \zeta_{i j}=J_{i j}+p_{j} y_{i}-p_{i} y_{j}, \quad \zeta_{4 i}=p_{i} y_{4}-p_{4} y_{j}-\frac{p^{k} J_{k i}}{\sqrt{2}\left(p_{0}-p_{4}\right)}, \\
& \zeta_{0 i}=-y_{i} p_{0}-\frac{p^{k} J_{k i}}{\sqrt{2}\left(p_{0}-p_{4}\right)}, \quad \zeta_{A}=p_{A}, \quad \zeta_{04}=-y_{4} p_{0},
\end{aligned}
$$

where we introduced $J_{a b}:=\frac{1}{2} S^{c d} \epsilon_{a b c d}$ as in the massive case, with the difference that now $J_{a 4}=0$, as a result of the constraints, meaning that we can work with a tensor $J_{i j}$ with $i, j \in$ $\{1,2,3\}$. We see that our general point is a function of the variables $\left\{p_{a}, y_{a}, J_{i j}\right\}$ including the four independent momentum variables $p_{a}$, four coordinates $y_{a}$ and three variables $J_{i j}$. Still, we have to impose (37). As a result, $J_{i j}$ has only 2 independent components, which gives in total 10 independent parameters for our coadjoint orbit.

As in the massive case, we can now deduce the expressions for the Poisson brackets on the coadjoint orbit by projecting the original Poisson bracket of $\mathfrak{g}^{*}$ on it. We find

$$
\begin{aligned}
& \left\{J_{k l}, J_{i j}\right\}=\delta_{k i} J_{l j}+\delta_{l j} J_{k i}-\delta_{k j} J_{l i}-\delta_{l i} J_{k j}, \quad\left\{y_{a}, p_{0}\right\}=-\frac{p_{a}}{p_{0}}, \\
& \left\{p_{a}, p_{b}\right\}=0, \quad\left\{y_{a}, y_{b}\right\}=0, \quad\left\{y_{a}, p_{b}\right\}=\delta_{a b}, \quad\left\{y_{a}, J_{j k}\right\}=0, \quad\left\{p_{a}, J_{i k}\right\}=0 .
\end{aligned}
$$

To quantise our system, we use the same approach as for a massive particle. In particular, one can introduce the same scalar product on the momentum space as in (28), and requiring hermiticity with respect to it fixes the form of the operator $\hat{y}_{a}$ as

$$
\hat{y}_{a}=i\left(-\frac{\partial}{\partial p^{a}}-\frac{p_{a}}{2 p^{0}}\right) \mathbb{I}
$$

while $J_{i j}$ is turned into the operator $\hat{J}_{i j}$ obeying the commutation relations of $s o(3)$, as is clear from the first equation of (43). We also have to replace $p_{0} y_{a}$ by $\frac{1}{2}\left(\hat{P}_{0} \hat{y}_{i}+\hat{y}_{i} \hat{P}_{0}\right)$ to consider the ordering of operators. In this framework, the momentum operators still take the form $\hat{P}_{A}=p_{A} \mathbb{I}$ as in the massive case while one can compute the generators of Lorentz group, which gives

$$
\begin{aligned}
& \hat{M}_{04}=i p_{0} \frac{\partial}{\partial p^{4}} \mathbb{I} \\
& \hat{M}_{i j}=\hat{J}_{i j}-i\left(p_{j} \frac{\partial}{\partial p^{i}}-p_{i} \frac{\partial}{\partial p^{j}}\right) \mathbb{I} \\
& \hat{M}_{0 i}=i p_{0} \frac{\partial}{\partial p^{i}} \mathbb{I}-\frac{p^{k} \hat{J}_{k i}}{p^{0}+p^{4}} \\
& \hat{M}_{4 i}=-i\left(-p_{4} \frac{\partial}{\partial p^{i}}+p_{i} \frac{\partial}{\partial p^{4}}\right) \mathbb{I}-\frac{p^{k} \hat{J}_{k i}}{p^{0}+p^{4}} .
\end{aligned}
$$

\section{Massive Case in Any Dimension}

In Minkowski spacetimes of arbitrary dimension $d>3$, massive particles are characterised by the mass-shell condition $P^{A} P_{A}=m^{2}$ and by additional mutually independent constraints obtained by fixing the eigenvalues of the other Casimir operators, whose number depends on the dimension of spacetime. These additional independent constraints generalise Equation (6) and fix a set of quantum numbers that play a role analogous to that of the spin in four dimensions. The total number of Casimir operators of $I S O(d-1,1)$ is equal to $\left\lfloor\frac{d+1}{2}\right\rfloor[25]$, which for a massive representation fixes the dimension of the coadjoint orbit to be

$$
\operatorname{dim} \mathcal{O}=\frac{d(d+1)}{2}-\left\lfloor\frac{d+1}{2}\right\rfloor .
$$


For $d=4$, we recover $\operatorname{dim} \mathcal{O}=8$, while for $d=5$, we recover $\operatorname{dim} \mathcal{O}=12$ and the dimension is always even, as it should be. One can identify a canonical point for the orbit of a massive particle following the same procedure as in Section 2:

$$
\bar{\zeta}_{A}=(m, \overrightarrow{0}), \quad \bar{\zeta}_{0 a}=0, \quad \bar{\zeta}_{b c}=\frac{1}{(d-3) !} S^{a_{1} \cdots a_{d-3}} \epsilon_{b c a_{1} \cdots a_{d-3}},
$$

where the components of the fully anti-symmetric tensor $S^{a_{1} \cdots a_{d-3}}$ have to obey the constraints that come from having fixed the eigenvalues of the higher-order Casimir operators. As detailed in Section 2 for the $d=5$ case, these constraints also fix the eigenvalues of all Casimir operators of the little group $S O(d-1)$ for a massive particle.

To identify the generic point on the orbit, we can also generalise the parametrisation of [16] as in Section 2. This means that we decompose an arbitrary element of the Lorentz group as $\Lambda=L R$, where $L$ is a boost and $R$ a rotation in $d$ spacetime dimensions. We also decompose a generic translation vector in terms of two $d$-dimensional vectors $z$ and $y$ satisfying the following properties:

$$
z=\left(z^{0}, \overrightarrow{0}\right), \quad y=(0, \vec{y}) .
$$

As a result, we find the following parameterisation for a generic point on the orbit:

$$
\begin{aligned}
& \zeta_{A}=\Lambda_{A}^{0} m:=p_{A}, \\
& \zeta_{0 a}=-p_{0} y_{a}+\frac{p^{e}}{(d-3) ! m} S^{b_{1} \cdots b_{d-3}} \epsilon_{a e b_{1} \cdots d-3}, \\
& \zeta_{a f}=\frac{1}{(d-3) !} S^{b_{1} \cdots b_{d}} \epsilon_{a f b_{1} \cdots b_{d-3}}+\frac{S^{b_{1} \cdots b_{d-3}} p^{e}}{m\left(m+p^{0}\right)(d-3) !}\left(\epsilon_{f b_{1} \cdots b_{d-3} e} p_{a}-\epsilon_{a b_{1} \cdots b_{d-3} e} p_{f}\right) .
\end{aligned}
$$

We can present this parameterisation in a more compact form by changing the variables,

$$
x_{a}=y_{a}-\frac{1}{(d-3) !} \frac{p^{c} S^{b_{1} \cdots b_{d-3}} \epsilon_{a c b_{1} \cdots b_{d-3}}}{m\left(m+p^{0}\right)} \text { and } J_{a c}=\frac{1}{(d-3) !} S^{b_{1} \cdots b_{d-3}} \epsilon_{a c b_{1} \cdots b_{d-3}},
$$

which leads to

$$
\zeta_{A}=\Lambda_{A}^{0} m:=p_{A}, \quad \zeta_{0 a}=-p_{0} x_{a}-\frac{p^{b} J_{a b}}{m+p^{0}}, \quad \zeta_{a b}=p_{b} x_{a}-p_{a} x_{b}+J_{a b} .
$$

A generic point is thus expressed as a function of the parameters $p_{a}, x_{a}, J_{a b}$ as in four and five dimensions. We have $d-1$ independent momentum variables, $d-1$ coordinate variables $x_{a}$ and the tensor $J_{a b}$ has a priori $\frac{(d-1)(d-2)}{2}$ independent components. However, we have to take into account the constraints on our coadjoint orbit: the mass-shell condition implies that only $d-1$ variables $p$ are independent, while the other constraints reduce the number of independent components of $J$ to $\frac{(d-1)(d-2)}{2}-\left\lfloor\frac{d-1}{2}\right\rfloor$. The Poisson brackets thus take the same form as in the case of $d=5$ displayed in (27), with the only change being that the indices $a, b, \ldots$ now run from 1 to $d-1$.

Following the same quantisation procedure as in Section 2, we promote the coordinates $\zeta$ 's on our phase space to operators in a Hilbert space. For this purpose, we introduce the inner product on scalar wave-functions in momentum space (and refer readers to the review [10] for the general case with non-zero spin),

$$
(f, g)=\int \frac{d^{d-1} p}{2 p_{0}} \overline{f(p)} g(p)
$$

which allows one to identify the Hermitian operators $\hat{x}_{a}$ corresponding to the variables $x_{a}$ :

$$
\hat{x}_{a}=i\left(-\frac{\partial}{\partial p^{a}}-\frac{i p_{a}}{2 p_{0}^{2}}\right) \text {. }
$$


The antisymmetric tensor $J_{a b}$ is promoted to an operator satisfying the $s o(d-1)$ algebra, meaning that we eventually obtain

$$
\hat{M}_{0 a}=-i p_{0} \frac{\partial}{\partial p^{a}} \mathbb{I}-\frac{p^{b} \hat{J}_{a b}}{m+p^{0}}, \quad \hat{M}_{a b}=-i\left(p_{b} \frac{\partial}{\partial p^{a}}-p_{a} \frac{\partial}{\partial p^{b}}\right) \mathbb{I}+\hat{J}_{a b} .
$$

\section{Massless Case in Any Dimension}

In order to generalise the analysis of five-dimensional massless particles of fixed helicity, we impose

$$
P_{A} P^{A}=0
$$

and we generalise the helicity condition (31b) as [9]

$$
P^{[A} \mathbb{W}^{\left.B_{1} \cdots B_{d-3}\right]}=0,
$$

where $\mathbb{W}^{A_{1} \cdots A_{d-3}}$ denotes the generalised Pauli-Lubanski tensor

$$
\mathbb{W}^{A_{1} \cdots A_{d-3}}=\frac{1}{2} \epsilon^{A_{1} \cdots A_{d-3} B C E} M_{B C} P_{E} .
$$

Notice that contracting (56) with $\epsilon_{A B_{1} \cdots B_{d-3} C D} M^{C D}$ one obtains $\mathbb{W}_{A_{1} \cdots A_{d-3}} \mathbb{W}^{A_{1} \cdots A_{d-3}}=0$ which is a condition that holds true for massless particles of discrete helicity in any dimension [10].

Working in the frame in which the standard $d$-momentum takes the form $\zeta_{A}=$ $(k, 0, \ldots, 0,-k)$, the helicity condition implies $\zeta_{i 0}=-\zeta_{i d-1}$. Drawing from [9], we can also generalise the spin Equation (6), obtaining conditions that involve the (higher-order) Casimir operators of the little group $S O(d-2)$ of a discrete-helicity massless particle:

$$
\begin{array}{ll}
\text { d odd }: & \mathbb{S}_{A}^{(n)}=c^{(n)} P_{A}, \quad 1 \leqslant n \leqslant \frac{d-3}{2}, \\
\underline{\text { d even }:} & \mathbb{S}_{A}^{(n)}=c^{(n)} P_{A} \quad \text { and } \quad \mathbb{W}_{A}^{\left(\frac{d}{2}-1\right)}=\lambda^{\left(\frac{d}{2}-1\right)} P_{A}, \quad 1 \leqslant n \leqslant \frac{d-4}{2},
\end{array}
$$

where

$$
\begin{aligned}
& \mathbb{S}_{A}^{(n)}=\frac{(-1)^{d}}{2(d-2 n-1) !} \epsilon_{E_{1} \cdots E_{d}} M^{E_{2} E_{3}} \cdots M^{E_{2 n} E_{2 n+1} \mathbb{W}^{(n)} E_{2 n+2} \cdots E_{d}}, \\
& \mathbb{W}_{A_{1} \cdots A_{d-2 n-1}}^{(n)}=\frac{1}{2^{n}} \epsilon_{A_{1} \cdots A_{d}} M^{A_{d-2 n} A_{d-2 n+1}} \cdots M^{A_{d-2} A_{d-1}} P^{A_{d}} .
\end{aligned}
$$

The real numbers $c^{(n)}$ denote the eigenvalues of the $S O(d-2)$ Casimir operatorseigenvalues that are in bijection with the Dynkin labels or the Young diagram labels corresponding to the irrep of $S O(d-2)$ that characterises the particle. The order- $2 n$ Casimir operator of $S O(d-2)$ reads as

$$
C^{(n)}=\frac{(2 n) !}{2^{n+1}} M^{i_{1} i_{2}} \cdots M^{i_{2 n-1} i_{2 n}} M_{\left[i_{1} i_{2}\right.} \cdots M_{\left.i_{2 n-1} i_{2 n}\right]},
$$

while for an even $d$, the real number $\lambda^{\left(\frac{d}{2}-1\right)}$ is the eigenvalue taken by the following operator [9]:

$$
\Lambda^{\left(\frac{d}{2}-1\right)}=-\frac{1}{2^{\frac{d}{2}-1}} \epsilon_{0 i_{1} \cdots i_{d-2} d-1} M^{i_{1} i_{2}} \cdots M^{i_{d-3} i_{d-2}} .
$$

To study massless coadjoint orbits with finite spin, we thus have to fix $P_{A} P^{A}=0$ and impose the helicity condition (56). We also have to specify a representation of the little group $S O(d-2)$ via Equation (58), which corresponds to fixing the eigenvalues of all Casimir operators of $S O(d-2)$. This fixes the dimensions of the coadjoint orbit as follows: 
the dimension of the Poincare group is $\frac{d(d+1)}{2}$, and there is one condition which comes from the mass-shell condition (55), together with $d-2$ constraints coming from the helicity condition (56) and $r=\left\lfloor\frac{d-2}{2}\right\rfloor$ constraints coming from (58). Eventually, we obtain

$$
\operatorname{dim} \mathcal{O}=\frac{d(d+1)}{2}-(1+d-2+r)
$$

where $r$ is the rank of $s o(d-2)$. For $d=4$ we find an orbit with 6 dimensions, while for $d=5$, we find an orbit with 10 dimensions, which agrees with the result discussed above (in the continuous-spin case for which the constraint (55) is still in order, we certainly cannot impose the helicity condition (56) since it would lead to $\mathbb{W}_{A_{1} \cdots A_{d-3}} \mathbb{W}^{A_{1} \cdots A_{d-3}}=0$ that does not apply for continuous-spin particles; see, e.g., [26,27] and the reviews [10,28]. Instead, we have to impose the constraint $\frac{1}{(d-3) !} \mathbb{W}_{A_{1} \cdots A_{d-3}} \mathbb{W} A_{1} \cdots A_{d-3}=\mu^{2}($ with $\mu \neq 0)$ together with $r$ extra constraints where $r=\left\lfloor\frac{d-3}{2}\right\rfloor$ is the rank of the short little group $S O(d-3)$ for a continuous-spin particle. These $r$ constraints read as in (58), except for the changes that have to be introduced in order to take the substitution $S O(d-2) \rightarrow S O(d-3)$ into account. As a result, for a continuous-spin particle, there are as many independent constraints as for a massive particle, which implies that the dimensions of the corresponding coadjoint orbits are the same. See the recent paper [29] and references therein for relevant works on continuous-spin particles in arbitrary dimensions).

Now, we have to determine our canonical point and, to this end, we can apply the same strategy as in $d=5$, obtaining

$$
\bar{\zeta}_{A}=(k, \ldots,-k), \quad \bar{\zeta}_{0 a}=0, \quad \bar{\zeta}_{a b}=\frac{1}{(d-3) !} S^{c_{1} \cdots c_{d-3}} \epsilon_{a b c_{1} \cdots c_{d-3}} .
$$

where the components of $S$ have to satisfy the constraints imposed by fixing the $S O(d-2)$ Casimirs. We also employ the same parameterisation as in $d=5$ for the Poincaré generators to find a generic point of the orbit. The matrices $B, D$ and $R$ take the following form in arbitrary dimensions:

$$
B=\left(\begin{array}{ccccc}
\Lambda^{+}{ }_{+} & 0 & \cdots & \cdots & 0 \\
\Lambda^{+}{ }_{-} & \frac{1}{\Lambda^{+}+} & \frac{\Lambda^{1}}{\Lambda^{+}} & \cdots & \frac{\Lambda^{d-2}+}{\Lambda_{+}} \\
\Lambda^{1}{ }_{+} & 0 & 1 & \cdots & 0 \\
\vdots & \vdots & \cdots & \ddots & 0 \\
\Lambda^{d-2}{ }_{+} & 0 & \cdots & 0 & 1
\end{array}\right), D=\left(\begin{array}{ccccc}
1 & \frac{\Lambda^{+}-}{\Lambda^{+}} & d_{1} & \cdots & d_{d-2} \\
0 & 1 & 0 & \cdots & 0 \\
0 & d_{1} & 1 & \cdots & 0 \\
\vdots & \vdots & \cdots & \ddots & 0 \\
0 & d_{d-2} & \cdots & 0 & 1
\end{array}\right), R=\left(\begin{array}{cc|c}
1 & 0 & 0_{d-2 \times d-2} \\
0 & 1 & \\
\hline 0_{d-2 \times d-2} & A_{d-2 \times d-2}
\end{array}\right)
$$

After acting on the canonical point and returning to Minkowski coordinates, we find the following parametrisation for a generic point of the orbit:

$$
\begin{aligned}
& \zeta_{i j}=J_{i j}+p_{j} y_{i}-p_{i} y_{j}, \quad \zeta_{d-1 i}=p_{i} y_{d-1}-p_{d-1} y_{i}-\frac{p^{k} J_{k i}}{\sqrt{2}\left(p_{0}-p_{d-1}\right)}, \\
& \zeta_{0 i}=-y_{i} p_{0}-\frac{p^{k} J_{k i}}{\sqrt{2}\left(p_{0}-p_{d-1}\right)}, \quad \zeta_{A}=p_{A},
\end{aligned}
$$

where $J_{i j}=\frac{1}{(d-3) !} S^{c_{1} \cdots c_{d-3}} \epsilon_{i j c_{1} \cdots c_{d-3}}$, while $i, j, k, \ldots \in\{1,2,3, \ldots, d-2\}$. The Poisson brackets thus take the same form as in the case of $d=5$ displayed in (43).

By applying the same steps as in the quantisation of a massive particle, one can also identify the operators corresponding to each variable on the orbit and obtain the following expressions for the Lorentz generators:

$$
\begin{aligned}
M_{0 d-1} & =i p_{0} \frac{\partial}{\partial p^{d-1}} \mathbb{I}, & M_{i j} & =\hat{J}_{i j}-i\left(p_{j} \frac{\partial}{\partial p^{i}}-p_{i} \frac{\partial}{\partial p^{j}}\right) \mathbb{I}, \\
M_{0 i} & =i p_{0} \frac{\partial}{\partial p^{i}} \mathbb{I}-\frac{p^{k} \hat{J}_{k i}}{p^{0}+p^{d-1}}, & M_{d-1 i} & =-i\left(-p_{d-1} \frac{\partial}{\partial p^{i}}+p_{i} \frac{\partial}{\partial p^{d-1}}\right) \mathbb{I}-\frac{p^{k} \hat{J}_{k i}}{p^{0}+p^{d-1}} .
\end{aligned}
$$




\section{Conclusions}

The coadjoint orbits of the four-dimensional Poincaré group $\operatorname{ISO}(3,1)$ associated with massive and massless particles with discrete spin were characterised in [13-16]. In this paper, we extended this characterisation to the Poincaré group $\operatorname{ISO}(d-1,1)$ of isometries of the Minkowski spacetime in arbitrary dimensions $d>3$. Following [14,15], for each type of particle, we first identified the constraints on the dual Lie algebra that define the coadjoint orbit, and then we selected a set of independent coordinates parametrising the orbit. We eventually checked that they define a non-degenerate symplectic manifold and recovered the Poincaré algebra via the canonical quantisation of the resulting phase-space variables. In analogy with the four-dimensional case, we stressed that massive particles correspond to generic coadjoint orbits-identified by specifying the values of all Casimir operators of the Poincaré algebra - while massless particles with discrete spin correspond to non-generic orbits of lower dimension. Let us stress that, when $d>5$, the effective little group $S O(d-2)$ of massless helicity-type particles has rank $r>1$. In these cases, we identified the additional constraints on the coadjoint orbit and explained how to recover the quantum numbers of the $S O(d-2)$ little group in a covariant manner with respect to the Lorentz group $S O(d-1,1)$. We also commented on how to identify the coadjoint orbits associated with continuous-spin particles and noticed that the orbits associated with such particles have the same dimension as those of massive particles, which is consistent with the possibility to recover the corresponding continuous-spin UIRs from massive ones with the limits of infinite spin and vanishing mass [30].

Author Contributions: Conceptualization, I.A.L., N.B. and A.C.; Formal analysis, I.A.L., N.B. and A.C.; Writing-original draft, I.A.L., N.B. and A.C. All authors contributed equally to this work. All authors have read and agreed to the published version of the manuscript.

Funding: This research was partially supported by the Fonds de la Recherche Scientifique-FNRS under Grants No. T.0022.19 (Fundamental issues in extended gravitational theories) and F.4503.20 (HighSpinSymm).

Institutional Review Board Statement: Not applicable.

Informed Consent Statement: Not applicable.

Data Availability Statement: No new data were created or analysed in this study.

Acknowledgments: We thank Glenn Barnich and Xavier Bekaert for discussions.

Conflicts of Interest: The authors declare no conflict of interest.

\section{References}

1. Wigner, E.P. On Unitary Representations of the Inhomogeneous Lorentz Group. Annals Math. 1939, 40, 149-204. [CrossRef]

2. Bargmann, V.; Wigner, E.P. Group Theoretical Discussion of Relativistic Wave Equations. Proc. Nat. Acad. Sci. USA 1948, 34, 211. [CrossRef]

3. Siegel, W.; Zwiebach, B. Gauge String Fields from the Light Cone. Nucl. Phys. B 1987, 282, 125-141. [CrossRef]

4. Siegel, W. Fields. arXiv 1999, arXiv:hep-th/9912205.

5. Binegar, B. Relativistic Field Theories in Three-dimensions. J. Math. Phys. 1982, 23, 1511-1517. [CrossRef]

6. Bekaert, X.; Boulanger, N. Tensor gauge fields in arbitrary representations of GL(D,R): Duality and Poincare lemma. Commun. Math. Phys. 2004, 245, 27-67. [CrossRef]

7. Bekaert, X.; Boulanger, N. On geometric equations and duality for free higher spins. Phys. Lett. B 2003, 561, 183-190. [CrossRef]

8. Bekaert, X.; Boulanger, N. Tensor gauge fields in arbitrary representations of GL(D,R). II. Quadratic actions. Commun. Math. Phys. 2007, 271, 723-773. [CrossRef]

9. Kuzenko, S.M.; Pindur, A.E. Massless particles in five and higher dimensions. Phys. Lett. B 2021, 812, 136020. [CrossRef]

10. Bekaert, X.; Boulanger, N. The Unitary representations of the Poincare group in any spacetime dimension. SciPost Phys. Lect. Notes 2021, 30, 47.

11. Kirillov, A.A. Lectures on the Orbit Method; Graduate Studies in Mathematics; American Mathematical Society: Providence, RI, USA, 2004.

12. Cushman, R.; van der Kallen, W. Adjoint and Coadjoint Orbits of the Poincaré Group. Acta Appl. Math. 2006, 90, 65-89. [CrossRef]

13. Carinena, J.F.; Gracia-Bondia, J.M.; Varilly, J.C. Relativistic Quantum Kinematics in the Moyal Representation. J. Phys. A 1990, $23,901$. [CrossRef] 
14. Andrzejewski, K.; Kijanka-Dec, A.; Kosiński, P.; Maslanka, P. Chiral fermions, massless particles and Poincare covariance. Phys. Lett. B 2015, 746, 417-423. [CrossRef]

15. Andrzejewski, K.; Gonera, C.; Goner, J.; Kosiński, P.; Maslanka, P. Spinning particles, coadjoint orbits and Hamiltonian formalism. arXiv 2020, arXiv:2008.09478.

16. Kosiński, P.; Maślanka, P. Relativistic Symmetries and Hamiltonian Formalism. Symmetry 2020, 12, 1810. [CrossRef]

17. Witten, E. Coadjoint Orbits of the Virasoro Group. Commun. Math. Phys. 1988, 114, 1. [CrossRef]

18. Barnich, G.; Oblak, B. Notes on the BMS group in three dimensions: I. Induced representations. J. High Energy Phys. 2014, 6, 129. [CrossRef]

19. Barnich, G.; Oblak, B. Notes on the BMS group in three dimensions: II. Coadjoint representation. J. High Energy Phys. 2015, 3, 033. [CrossRef]

20. Oblak, B. BMS Particles in Three Dimensions; Springer: Berlin/Heidelberg, Germany, 2017; p. 476.

21. Balog, J.; Feher, L.; Palla, L. Coadjoint orbits of the Virasoro algebra and the global Liouville equation. Int. J. Mod. Phys. A 1998, 13, 315-362. [CrossRef]

22. Raeymaekers, J. Quantization of conical spaces in 3D gravity. J. High Energy Phys. 2015, 3, 060. [CrossRef]

23. Barnich, G.; Ruzziconi, R. Coadjoint representation of the BMS group on celestial Riemann surfaces. J. High Energy Phys. 2021, 6, 079. [CrossRef]

24. Marsden, J.; Ratiu, T. Introduction to Mechanics and Symmetry; Springer: Berlin/Heidelberg, Germany, 1999.

25. Chaichian, M.; Demichev, A.P.; Nelipa, N.F. The Casimir Operators of Inhomogeneous Groups. Commun. Math. Phys. 1983, 90, 353. [CrossRef]

26. Brink, L.; Khan, A.; Ramond, P.; Xiong, X.z. Continuous spin representations of the Poincare and super-Poincare groups. J. Math Phys. 2002, 43, 6279. [CrossRef]

27. Bekaert, X.; Mourad, J. The Continuous spin limit of higher spin field equations. J. High Energy Phys. 2006, 1, 115. [CrossRef]

28. Bekaert, X.; Skvortsov, E.D. Elementary particles with continuous spin. Int. J. Mod. Phys. A 2017, 32, 1730019. [CrossRef]

29. Metsaev, R.R. Mixed-symmetry continuous-spin fields in flat and AdS spaces. Phys. Lett. B 2021, 820, 136497. [CrossRef]

30. Khan, A.; Ramond, P. Continuous spin representations from group contraction. J. Math. Phys. 2005, 46, 053515; Erratum: J. Math. Phys. 2005, 46, 079901. [CrossRef] 